\title{
Intracrystalline Diffusion in Zeolites Studied by Neutron Scattering Techniques
}

\author{
H. Jobic ${ }^{1}$ and A. Méthivier ${ }^{2}$ \\ 1 Institut de Recherches sur la Catalyse, CNRS, 2 avenue A. Einstein, 69626 Villeurbanne Cedex - France, \\ 2 Institut français du pétrole, BP 3, 69390 Vernaison - France \\ e-mail: jobic@catalyse.cnrs.fr - alain.methivier@ifp.fr
}

Résumé - Étude de la diffusion intracristalline dans les zéolithes par diffusion des neutrons — Les techniques de diffusion neutronique permettent d'étudier les différents mouvements des molécules adsorbées dans les zéolithes : vibrations, rotations et translation. En utilisant différents instruments, il est maintenant possible de mesurer des coefficients de diffusion dans la gamme $10^{-7}-10^{-14} \mathrm{~m}^{2} \mathrm{~s}^{-1}$. En outre, la diffusion incohérente, provenant de molécules hydrogénées, donne accès à l'auto-diffusion ; alors que la diffusion cohérente, en utilisant des molécules deutérées, permet d'obtenir le coefficient de diffusion de transport. Plusieurs applications des méthodes neutroniques sont décrites dans cette revue. Elles sont illustrées par différents exemples : xylènes dans des zéolithes de type $\mathrm{X}$, alcanes dans des zéolithes de type MFI, n-alcanes dans la 5A, et eau dans des zéolithes de type A et X.

\footnotetext{
Abstract - Intracrystalline Diffusion in Zeolites Studied by Neutron Scattering Techniques Neutron scattering techniques can be used to study the different motions of molecules adsorbed in zeolites: vibrations, rotations and translation. Using combined instruments, it is now possible to measure diffusivities ranging from $10^{-7}$ to $10^{-14} \mathrm{~m}^{2} \mathrm{~s}^{-1}$. Furthermore, incoherent scattering, using hydrogenated molecules, allows to determine the self-diffusivity; while coherent scattering, studying deuterated molecules, gives access to the transport diffusivity. Several applications of the neutron techniques are described in this review. They are illustrated by the case study of xylenes in X-type zeolites. Other examples are related to alkanes in MFI-type zeolites, $n$-alkanes in 5A zeolite, and water in X and A-type zeolites.
} 


\section{INTRODUCTION}

Adsorption separation processes are key operations in the field of refining, petrochemical and natural gas treatment. These operations are based on selective adsorption of hydrocarbon molecules or impurities in adsorbent materials. In this respect, the molecular sieving properties of zeolites are frequently used. The performances of these materials are determined by their thermodynamic as well as their transport properties. The transport properties must be controlled as diffusion must be as fast as possible in order to reduce the adsorbent inventory. Moreover, some separations are based on the differences between the diffusion coefficients of the various components. Thus, intracrystalline diffusion, i.e. selfand transport diffusion, of hydrocarbon molecules in zeolites must be controlled.

It is well admitted that the pore size and shape has a drastic effect on molecular diffusion in zeolites, however, measuring diffusion coefficients in these microscopic materials is not an easy task. Recent comparisons between the results obtained from different techniques still show discrepancies of several orders of magnitude. There is usually a reasonable agreement between incoherent quasi-elastic neutron scattering (QENS) and pulsed-field gradient NMR (PFG NMR) techniques which measure the self-diffusivity at thermodynamic equilibrium. QENS is a truly microscopic method since one can obtain information on the diffusion mechanism at the atomic level. PFG NMR is also classified as a microscopic method because diffusion is followed in the interior of a zeolite crystal. Macroscopic methods are related to diffusion studies over a zeolite bed.

Transport diffusivities are traditionally obtained from macroscopic measurements, under non-equilibrium conditions. However, it has recently been shown that transport diffusivities can be measured by coherent QENS, at equilibrium $[1,2]$. It may seem surprising to derive a non-equilibrium quantity from QENS experiments performed at equilibrium. The explanation was given by Onsager in the thirties, when he formulated his regression hypothesis. This was later on formalized through the linear response theory [3]. The possibility to derive a transport diffusivity from density fluctuations, around equilibrium, has been used recently in molecular dynamics simulations [4].

During a QENS experiment, one probes molecular diffusion over space scales ranging from a few $\AA$ to several $\mathrm{nm}$. At short distances, jumps between adsorption sites can be observed, so that the characteristic lengths and times of the elementary steps can be determined using jump diffusion models. Such jump processes can be modelled by simulation techniques, which makes comparisons between QENS and simulations very fruitful. Over space scales of a few unit cells, Fickian diffusion is observed so that the diffusion coefficients can be derived without a model.
Apart from diffusion, neutron scattering techniques have also the potential to probe the other molecular motions of vibration and rotation. Inelastic neutron scattering (INS) has been used to measure the vibrational modes of zeolite frameworks and of adsorbed molecules. Both the geometry and the dynamics of the rotational motions can be characterized by selecting the proper time window in QENS experiments.

The application of these techniques to the study of complex systems can provide good insights into the transport and mobility phenomena of adsorbed molecules. In this paper, we report examples devoted to diffusion mechanism in various hydrocarbon/zeolite and water/zeolite systems which are all of industrial relevance for separation applications.

These systems are: xylenes in X-type zeolites, alkanes in MFI-type zeolites, $n$-alkanes in A-type zeolite, water in X and A-type zeolites.

\section{EXPERIMENTAL}

The production of intense beams of neutrons requires large reactor — or accelerator-based neutron sources. A complex system of guides and advanced instruments has to be designed to exploit the full power of these costly facilities. At the time being, Europe has still the lead in neutron science. The two main sources are:

- the high-flux reactor at the Institut Laue-Langevin (ILL), in Grenoble (F);

- the ISIS spallation source at the Rutherford Laboratory (UK).

Some competition is expected in the future: the USA are building a spallation source at Oak Ridge, and Japan is building a similar facility in Tokai (the power level should rise progressively in both places starting in 2006, full power being attained in 2010 or so).

At least until the end of the decade, and possibly even longer, the ILL will stay unique in that a very wide range of energy transfers is accessible using combined instruments (Fig. 1). The range $1-500 \mathrm{meV}\left(1 \mathrm{meV} \approx 8 \mathrm{~cm}^{-1}\right)$ can be covered using time-of-flight (TOF) and Beryllium filter (BeF) spectrometers, allowing to study vibrational modes of adsorbed molecules. Depending on their timescales, the rotational and translational motions can be characterized on TOF, back-scattering (BS), and neutron spin-echo (NSE) instruments. The energy transfers involved in QENS are usually $<1 \mathrm{meV}$. By varying the instrumental resolution, characteristic times ranging from $10^{-8}$ to $10^{-12} \mathrm{~s}$ can be accessed. It has been shown recently that the NSE method extends this timescale window to slower motions by two orders of magnitude. A spin-echo set-up exists at the Laboratoire Léon Brillouin (LLB, France), but there is only one NSE instrument in the world, at the ILL, which has the capacity to reach times of several hundreds of ns, the equivalent energy resolution being of a few neV. 


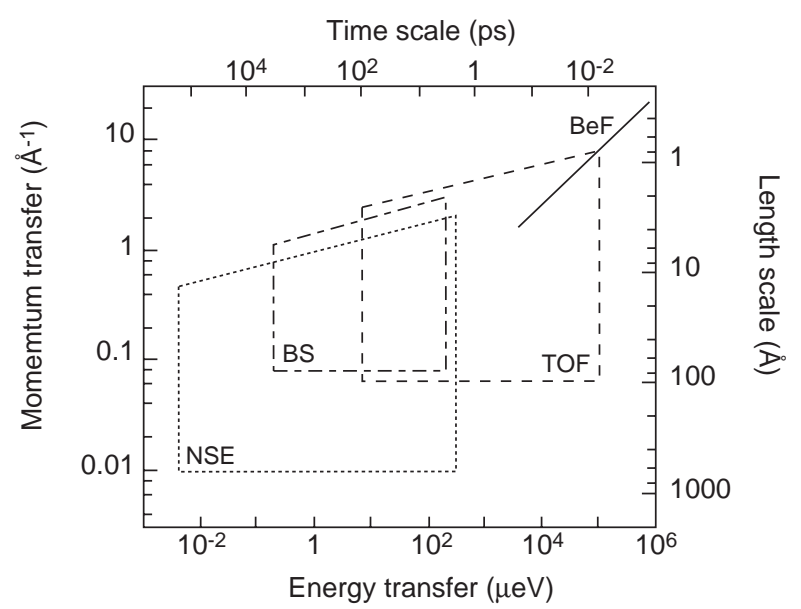

Figure 1

Combined neutron instrumentation at the ILL: neutron spin-echo (NSE), back-scattering (BS), time-of-flight (TOF), and beryllium-filter $(\mathrm{BeF})$. The $(\mathrm{Q}, \mathrm{E})$ window on the $\mathrm{BeF}$ filter spectrometer is so narrow that it falls on a single line. The accessible energy and momentum transfers ranges are shown along with their corresponding time and length scales.

\section{INTRODUCTION TO THE THEORY OF NEUTRON SCATTERING}

\subsection{Scattering Theory, Neutron Cross-Sections}

Neutrons interact with nuclei via very short range $(\approx \mathrm{fm})$ nuclear forces. A neutron has both particle-like and wavelike properties: it has zero charge, a $\operatorname{spin}^{1 / 2}$, a mass slightly larger than the mass of a proton, and a wavelength $\lambda$ [5]. Since $\lambda$ is a scalar, one uses the wave vector $\mathbf{k}$ in scattering theory (the magnitude of $\mathbf{k}$ is $2 \pi / \lambda$ ).

The scattering vector is defined by:

$$
\mathbf{Q}=\mathbf{k}_{0}-\mathbf{k}_{1}
$$

where $\mathbf{k}_{0}$ and $\mathbf{k}_{1}$ are, respectively the incident and final wave vectors. The momentum which is transferred to the sample is $\hbar \mathbf{Q}$. When there is an exchange of energy between the neutron and the sample, the energy transfer is defined as:

$$
\hbar \omega=E_{0}-E_{1}
$$

where $E_{0}$ and $E_{1}$ are, respectively the incident and scattered neutron energies. In most QENS experiments, one measures the double-differential cross-section, $d^{2} \sigma / d \Omega d E$, which represents the number of neutrons scattered into the solid angle $d \Omega$ with energy in the range $d E$. The amplitude, $b$, of the scattered wave varies between nuclei (because of different isotopes or spins), so that averages have to be performed for each element. The total cross-section per scatterer is obtained by integrating over energies and solid angles:

$$
\sigma=\int d \Omega \frac{d \sigma}{d \Omega}=\int d E \int d \Omega \frac{d^{2} \sigma}{d \Omega d E}=4 \pi<b^{2}>
$$

The scattering cross-section can be split into coherent and incoherent contributions (noting that the average of the square is different from the square of the average). The coherent elastic scattering has a phase term in $\exp (i \mathbf{Q} \cdot \mathbf{R})$, and thus takes into account interference effects. The coherent scattering cross section is:

$$
\sigma_{c o h}=4 \pi<b>^{2}
$$

The incoherent part, which has no phase relationship, corresponds to the difference between the total and the coherent cross-sections:

$$
\sigma_{i n c}=4 \pi\left(<b^{2}>-<b>^{2}\right) .
$$

it therefore represents the mean square deviation from the mean potential.

Table 1 lists the different cross-sections for some atoms. The absorption of neutrons by matter is small for most elements so that neutrons are highly penetrating and can be used in complex sample environments such as furnaces, cryostats, and pressure cells. The few elements which absorb neutrons are used for protection (e.g. Cd) or for detection (e.g. B).

The cross-section represents the area of a nucleus as seen by a neutron, it is usually measured in barns ( 1 barn = $10^{-24} \mathrm{~cm}^{2}$ ). It appears from Table 1 that there is no systematic variation of the cross-section across the periodic table, as it is the case for X-ray scattering. Hydrogen has the largest crosssection. This explains why the first measurements performed on diffusion in zeolites used hydrogenated molecules, in order to obtain the best contrast with the signal from the zeolite. Since the cross-section of hydrogen is incoherent, it gives access to the individual motions of the molecules inside the porous network, and thus to the self-diffusivity. The coherent cross-sections give rise to Bragg scattering for the framework (Al, Si, O, P), and to the collective mobility of sorbates. For coherent scatterers such as $\mathrm{N}_{2}, \mathrm{O}_{2}, \mathrm{CO}_{2}$, deuterated molecules, etc., diffusing in a porous material, one can derive the transport diffusivity (v.i.).

The measured intensity can be split into coherent and incoherent contributions, as in the case of the scattering cross-sections:

$$
\frac{d^{2} \sigma}{d \Omega d E}=\frac{k_{1}}{k_{0}} \frac{N}{4 \pi \hbar}\left[\sigma_{c o h} S_{c o h}(Q, \omega)+\sigma_{i n c} S_{i n c}(Q, \omega)\right]
$$

where $S(Q, \omega)$ is called the scattering function, or dynamical structure factor. Integrating over energy yields the structure factor, $S(Q)$. For incoherent scattering, the intensity is 
TABLE 1

Coherent, incoherent, and absorption cross-sections for some elements. They are given in barns $\left(1\right.$ barn $\left.=10^{-24} \mathrm{~cm}^{2}\right)$. The absorption cross-section is proportional to the neutron wavelength, here $\lambda=1 \AA$.

The nuclear spins are given for some isotopes, as well as their relative abundance (\%).

\begin{tabular}{|c|c|c|c|c|c|c|}
\hline Element & Atomic number & $\begin{array}{c}\text { Mass number } \\
\text { (natural abundance) }\end{array}$ & Spin & $\sigma_{\mathrm{coh}}$ & $\sigma_{\mathrm{inc}}$ & $\sigma_{\mathrm{abs}}$ \\
\hline $\mathrm{H}$ & 1 & $\begin{array}{l}1(99.985) \\
2(0.015)\end{array}$ & $\begin{array}{c}1 / 2 \\
1\end{array}$ & $\begin{array}{c}1.7568 \\
1.7583 \\
5.592\end{array}$ & $\begin{array}{c}80.26 \\
80.27 \\
2.05\end{array}$ & $\begin{array}{c}0.19 \\
0.19 \\
0\end{array}$ \\
\hline $\mathrm{He}$ & 2 & $\begin{array}{l}3(0.00014) \\
4(99.9998)\end{array}$ & $\begin{array}{c}1 / 2 \\
0\end{array}$ & $\begin{array}{l}1.34 \\
4.42 \\
1.34\end{array}$ & $\begin{array}{c}0 \\
1.6 \\
0\end{array}$ & $\begin{array}{c}0.0004 \\
2963 \\
0\end{array}$ \\
\hline $\mathrm{B}$ & 5 & & & 3.54 & 1.7 & 426 \\
\hline $\mathrm{C}$ & 6 & $\begin{array}{c}12(98.9) \\
13(1.1)\end{array}$ & $\begin{array}{c}0 \\
1 / 2 \\
\end{array}$ & $\begin{array}{c}5.551 \\
5.559 \\
4.81 \\
\end{array}$ & $\begin{array}{c}0.001 \\
0 \\
0.034\end{array}$ & $\begin{array}{l}0 \\
0 \\
0\end{array}$ \\
\hline $\mathrm{N}$ & 7 & $\begin{array}{c}14(99.63) \\
15(0.37)\end{array}$ & $\begin{array}{c}1 \\
1 / 2\end{array}$ & $\begin{array}{c}11.01 \\
11.03 \\
5.21\end{array}$ & $\begin{array}{c}0.5 \\
0.5 \\
0\end{array}$ & $\begin{array}{c}1.1 \\
1.1 \\
0\end{array}$ \\
\hline $\mathrm{O}$ & 8 & & & 4.232 & 0 & 0 \\
\hline $\mathrm{F}$ & 9 & $19(100)$ & $1 / 2$ & 4.017 & 0.0008 & 0.005 \\
\hline $\mathrm{Ne}$ & 10 & & & 2.62 & 0.008 & 0.022 \\
\hline $\mathrm{Al}$ & 13 & $27(100)$ & $5 / 2$ & 1.495 & 0.008 & 0.13 \\
\hline $\mathrm{Si}$ & 14 & & & 2.163 & 0.004 & 0.1 \\
\hline $\mathrm{P}$ & 15 & $31(100)$ & $1 / 2$ & 3.307 & 0.005 & 0.1 \\
\hline $\mathrm{S}$ & 16 & 32 & & 1.02 & 0.007 & 0.29 \\
\hline $\mathrm{Cl}$ & 17 & & & 11.531 & 5.2 & 18.6 \\
\hline $\mathrm{Ar}$ & 18 & $\begin{array}{c}36(0.337) \\
38(0.063) \\
40(99.6)\end{array}$ & $\begin{array}{l}0 \\
0 \\
0\end{array}$ & $\begin{array}{c}0.458 \\
77.9 \\
1.5 \\
0.42\end{array}$ & $\begin{array}{c}0.225 \\
0 \\
0 \\
0\end{array}$ & $\begin{array}{l}0.38 \\
2.89 \\
0.44 \\
0.37\end{array}$ \\
\hline $\mathrm{V}$ & 23 & & & 0.018 & 5.08 & 2.82 \\
\hline $\mathrm{Kr}$ & 36 & & & 7.65 & 0.03 & 13.9 \\
\hline $\mathrm{Cd}$ & 48 & & & 3.04 & 3.46 & 1400 \\
\hline $\mathrm{Xe}$ & 54 & $\begin{array}{l}129(26.4) \\
131(21.2) \\
132(26.9)\end{array}$ & $\begin{array}{c}1 / 2 \\
3 / 2 \\
0\end{array}$ & $\begin{array}{c}2.96 \\
- \\
- \\
-\end{array}$ & $\begin{array}{l}0 \\
- \\
- \\
0\end{array}$ & $\begin{array}{c}13.28 \\
11.67 \\
47.22 \\
0.25\end{array}$ \\
\hline $\mathrm{Pt}$ & 78 & & & 11.58 & 0.13 & 5.72 \\
\hline
\end{tabular}

smoothly decaying with $Q$, but for coherent scattering one has a maximum related to the intermolecular distance [6]. For data fitting, it is easier to work with the scattering function than with the double-differential cross-section, since there are no experimental parameters.

For a molecule, the usual approximation is to consider that the long-range translations, the rotations, and the vibrations are not coupled. The coherent scattering function is treated elsewhere [7], the incoherent scattering function will be mainly discussed here. It can then be written as a convolution product of the different scattering functions:

$$
S_{i n c}(Q, \omega)=S_{i n c}^{\text {trans }}(Q, \omega) \otimes S_{i n c}^{r o t}(Q, \omega) \otimes S_{i n c}^{v i b}(Q, \omega)
$$

The intramolecular vibrations occur typically in the energy range $10-400 \mathrm{meV}$. The neutron gains or loses energy by interacting with discrete vibrational levels in the sample. The technique is called inelastic neutron scattering (INS). The scattering function can be calculated in several ways:

- from ab initio quantum calculations [8];

- from molecular dynamic simulations [9];

- from the Wilson's GF matrix method [10].

This last method has been used for a preliminary analysis of the INS data obtained for hydrogenated xylenes in X-type zeolites (see paragraph 3.1). For a polyatomic molecule, the molecular vibrations are resolved into normal modes. In neutron energy loss, the INS spectrum will consist in $\delta$ functions 
at the frequencies $\omega_{\lambda}$ corresponding to the normal modes $\lambda$. At low temperature, the scattering function corresponding to a fundamental will be given by:

$$
S(\mathbf{Q}, \omega)=\sum_{d} \exp \left(-\mathbf{Q}^{2}<\mathbf{u}_{d}{ }^{2}>\right) \frac{\hbar\left|\mathbf{Q} \cdot \mathbf{C}_{d}^{\lambda}\right|^{2}}{2 m_{d} \omega_{\lambda}} \delta\left(\omega-\omega_{\lambda}\right)
$$

The intensity is mainly governed by the product $\left|\mathbf{Q} \cdot \mathbf{C}_{d}^{\lambda}\right|^{2}$, where the vectors $\mathbf{C}_{d}^{\lambda}$ describe the displacement of the $d$ th atom during the $\lambda$ th normal mode (a powder average has to be performed to take into account all possible orientations of $\mathbf{C}$ relative to $\mathbf{Q})$. The factor $\exp \left(-\mathbf{Q}^{2}\left\langle\mathbf{u}_{d}^{2}\right\rangle\right)$, where $\left\langle\mathbf{u}_{d}^{2}\right\rangle$ is the mean-square amplitude for an atom $d$, is called the Debye-Waller factor.

The rotational and translational motions generally imply smaller energy transfers, and the technique is called quasielastic because the spectra are centred around zero energy transfer. During a normal QENS experiment, one measures in $\mathbf{Q}-\omega$ space motions which occur in real space and in time (Fig. 2). The scattering functions are the space and time Fourier transforms of a correlation function $G(\mathbf{r}, t)[11]$

$$
S(\mathbf{Q}, \omega)=\frac{1}{2 \pi} \int d t \exp (-i \omega t) \int d \mathbf{r} \exp (i \mathbf{Q} \cdot \mathbf{r}) G(\mathbf{r}, t)
$$

With incoherent scattering, one follows the motions of individual atoms or molecules and it is the self-correlation function, $G_{S}(\mathbf{r}, t)$, which is probed in Eq. (9). This function corresponds to the probability of finding an atom at position $\mathbf{r}$ at time $t$ if the same atom was at the origin at time zero. With coherent scattering, one probes the full correlation function, $G(\mathbf{r}, t)$. Here, one considers the relative motions of the atoms as a function of time. If one atom is at the origin at time zero, $G(\mathbf{r}, t)$ gives the probability of finding any atom (including the same) at position $\mathbf{r}$ at time $t$.

For rotations, the incoherent scattering function can be separated into an elastic component and a quasi-elastic contribution:

$$
S_{\text {inc }}^{r o t}(\mathbf{Q}, \omega)=A_{0}(\mathbf{Q}) \delta(\omega)+\sum_{\ell} A_{\ell}(\mathbf{Q}) \Lambda_{\ell}
$$

An elastic component occurs because the self-correlation function has a finite value at long times. This happens for example if an atom is rotating around a fixed point. The factor $A_{0}(\mathbf{Q})$, which governs the intensity of the $\delta$ function is called the elastic incoherent structure factor (EISF). This factor corresponds to the space Fourier transform of the proton trajectory averaged over the timescale of the experiment [5]. The variation of the EISF with $Q$ gives information on the geometry of rotation. The experimental EISF can be compared with various models, e.g. rotational diffusion on a sphere, diffusion within a sphere, uniaxial rotation, and fluctuations around equilibrium positions. Characteristic times of the rotational motions can be obtained from the quasielastic component in Eq. 10, which consists of a sum of Lorentzians $\Lambda_{\ell}$.

In zeolites, translations are slower than rotations and the motion has to be followed over a length scale that is much larger than the cage-to-cage distance. Slow diffusivities imply long times in $G(\mathbf{r}, t)$ and will therefore correspond to small $\omega$. The long wavelengths, involving averages over large distances will correspond to small $Q$. The scattering functions for diffusion are described in detail in paragraph 2.2 Schematic representations of QENS spectra are shown in Figures 2a and 2b. When the characteristic time, $\tau$, of the dynamical process to be measured is shorter than the inverse of the instrumental resolution, $\Delta \omega$, a broadening of the elastic peak will be observed (Fig. $2 b$ ), allowing to derive a diffusion coefficient from the spectra obtained at various $Q$

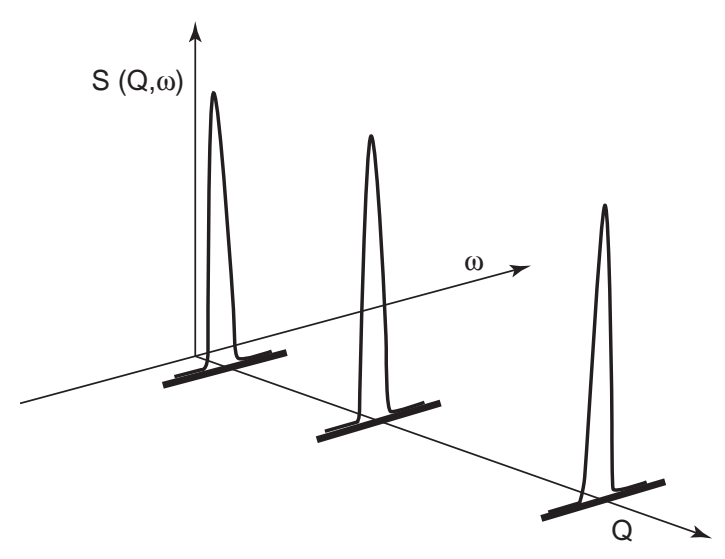

a)

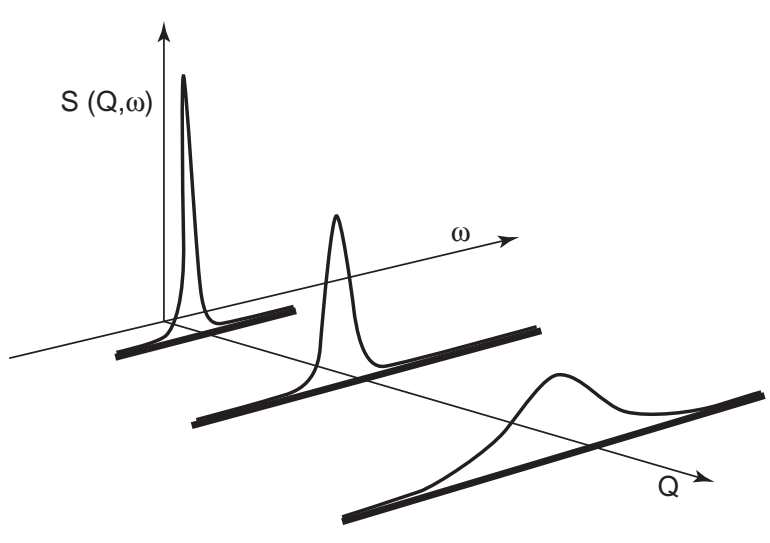

b)

Figure 2

Schematic representations of scattering functions in the case of a) elastic scattering, and b) quasi-elastic scattering. 
values. On the other hand, when $\tau$ is larger than $1 / \Delta \omega$, the shape is given by the instrumental resolution (Fig. 2a). In this case, the diffusivity cannot be extracted from the measurements, and a higher energy resolution is required to probe longer times. If the motion is too slow to be measured on TOF or BS spectrometers (i.e. the broadening is too small), then the diffusion may be accessible by neutron spin echo (NSE).

NSE is another neutron technique from which the Fourier transform of the scattering function, Eq. (9), is obtained:

$$
I(\mathbf{Q}, t)=\int d \omega \exp (i \omega t) S(\mathbf{Q}, \omega)
$$

$I(\mathbf{Q}, t)$ is called the intermediate scattering function since it is defined by:

$$
I(\mathbf{Q}, t)=\int d r \exp (i \mathbf{Q} \cdot \mathbf{r}) G(\mathbf{r}, t)
$$

so that the scattering function corresponds to:

$$
S(\mathbf{Q}, \omega)=\frac{1}{2 \pi} \int d t \exp (-i \omega t) I(\mathbf{Q}, t)
$$

With the NSE method, data are not recorded as a function of energy transfer, but as a function of time. Elastic and quasi-elastic representations are shown in Figures $3 a$ and $3 b$, respectively. The normalisation of $I(\mathbf{Q}, t)$ is done using $I(\mathbf{Q}, 0)$ which corresponds to the structure factor, $S(\mathbf{Q})$ :

$$
I(\mathbf{Q}, 0)=\int d \omega S(\mathbf{Q}, \omega)=S(\mathbf{Q})
$$

It can be noted that the problem of instrumental resolution, which is determined experimentally using an elastic scatterer, is easier to cope with in NSE than in TOF or BS techniques.
Energy spectra, (Fig. 2b), are convoluted with the instrumental resolution. In the time domain, the data obtained with the sample have simply to be divided by the reference scans obtained with an elastic scatterer. This corresponds to a deconvolution of the instrumental resolution in the energy domain. Another characteristic of the NSE technique is that deuterated molecules or coherent scatterers are preferentially studied, this is because the beam polarisation is reduced if the sample contains hydrogen atoms [12].

\subsection{Derivation of Self- and Transport Diffusivities}

Incoherent scattering allows to determine the self-diffusivity, $D_{S}$, whereas coherent scattering gives access to the transport diffusivity, $D_{t}$. The experiments are always performed at equilibrium. When the scattering is both incoherent and coherent, then both diffusivities can in principle be determined simultaneously.

Let us examine first the incoherent scattering. The motion of a given atom (or single molecule) is represented by the diffusion equation (Fick's second law):

$$
\frac{\partial p(\mathbf{r}, t)}{\partial t}=D_{s} \nabla^{2} p(\mathbf{r}, t)
$$

where $D_{S}$ is the self-diffusion coefficient. The self-correlation function, $G_{S}(\mathbf{r}, t)$, is an appropriate solution for $p(\mathbf{r}, t)$, the probability to find the atom (or molecule) at position $\mathbf{r}$ at time $t$. If the atom is at the origin at time zero, $G_{S}(\mathbf{r}, 0)=\delta(\mathbf{r})$, and one has a Gaussian expression for the self-correlation function:

$$
G_{s}(\mathbf{r}, t)=\frac{1}{\left(4 \pi D_{s} t\right)^{3 / 2}} \exp \left(-\frac{r^{2}}{4 D_{s} t}\right)
$$
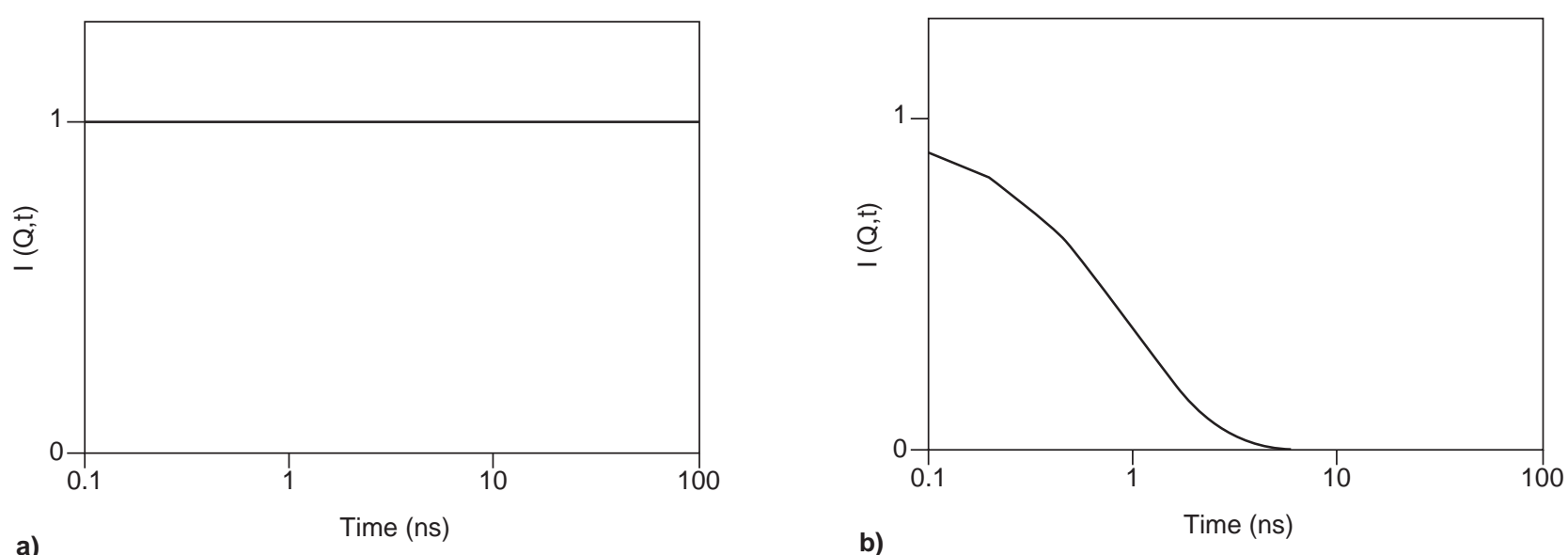

Figure 3

Schematic representations of the intermediate scattering function, for one $Q$ value, in the case of a) elastic scattering, and b) quasi-elastic scattering. 
The scattering function, Eq. (9), can then be calculated. As a first step, the spatial Fourier transform gives the intermediate self-scattering function:

$$
I_{s}(\mathbf{Q}, t)=\exp \left(-D_{s} Q^{2} t\right)
$$

The incoherent scattering function is obtained from the time Fourier transform of the intermediate self-scattering function:

$$
S_{i n c}(\mathbf{Q}, \omega)=\frac{1}{\pi} \frac{D_{s} Q^{2}}{\omega^{2}+\left(D_{s} Q^{2}\right)^{2}}
$$

Eq. (18) corresponds to an isotropic diffusion. Other analytical expressions can be derived for anisotropic diffusion (1-D, 2-D) or for single-file diffusion. Experimentally, energy spectra are measured at different scattering angles, corresponding to different values of the scattering vector $Q$ (Fig. 2b). According to Eq. 18, one should obtain a Lorentzian curve, with a half-width at half-maximum $(H W H M)$ :

$$
\Delta \omega(Q)_{i n c}=D_{s} Q^{2}
$$

It should be noted that the above expressions are only valid at large distances, corresponding to small $Q$ values. At larger $Q$ values, there is a deviation from the linear variation between $\Delta \omega$ and $Q^{2}$. Details of the elementary diffusive steps can then be obtained using jump diffusion models [13].

For coherent scattering, the scattering function depends on the total correlation function, $G(\mathbf{r}, t)$. This function gives the correlation between the densities at two different positions and different times:

$$
G(\mathbf{r}, t)=\frac{1}{\rho}\langle\rho(0,0) \rho(\mathbf{r}, t)\rangle
$$

where $\rho$ is the macroscopic number density. The transport diffusivity, $D_{t}$, can be obtained from QENS experiments because one is following the evolution of local concentration gradients around equilibrium. At low $Q$ values, the coherent scattering function for an isotropic motion has the form [14]:

$$
S_{c o h}(\mathbf{Q}, \omega)=\frac{S(Q)}{\pi} \frac{D_{t} Q^{2}}{\omega^{2}+\left(D_{t} Q^{2}\right)^{2}}
$$

The first difference with incoherent scattering is that the intensity varies as $S(Q)$, leaving aside the influence of the Debye-Waller factor. One therefore expects a non-monotonic variation of the total intensity as a function of $Q$. In the small $Q$ domain, the line-shape of the coherent scattering function is still Lorentzian, with a $H W H M$ :

$$
\Delta \omega(Q)_{c o h}=D_{t} Q^{2}
$$

Coherent neutron scattering is in general more complicated than incoherent scattering. However, for a totally coherent scatterer such as $\mathrm{CF}_{4}$, the rotational contribution is negligible because of the symmetry of the molecule [2]. A surprising conclusion is that it is easier to fit the spectra obtained for $\mathrm{CF}_{4}$ than for $\mathrm{CH}_{4}$.

\section{APPLICATIONS}

Using the various neutron spectrometers described in the Experimental section, one has the possibility to characterize the different molecular motions of the adsorbed molecules: vibrations, rotations, and translations. This will be illustrated by the case study of xylene isomers in X-type zeolites. Other examples related to diffusion will concern alkanes in MFI zeolite, $n$-alkanes in 5A zeolite, and water in $\mathrm{X}$ and A-type zeolites.

\subsection{Xylenes in X-Type Zeolites}

The para-xylene market has enjoyed high growth rates over the years and it is expected that this trend will continue for the future. Xylenes are produced from catalytic reforming of naphta. Isomerisation combined with separation allows to produce high purity para-xylene in agreement with the market demand. For this reason, the separation of para-xylene is a key operation which requires high purity, high yield, and high capacity. Adsorption is widely used to perform the separation, because of it's high efficiency. The adsorbents which are used for selective adsorption of para-xylene are $\mathrm{X}$ or $\mathrm{Y}$ zeolites exchanged with adequate cations. Liquid phase Simulated Counter Current adsorption is mainly used as it is the most efficient process [15].

The primary requirement for an economic separation process is an adsorbent with sufficiently high selectivity, high capacity, as well as high mass transfer rates. These separation properties are strongly dependent upon the zeolite parameters as depicted by numerous studies.

- The first parameter which can influence the adsorption behaviour is the compensating cation. For example, NaY is selective for meta-xylene and $\mathrm{KY}$ is selective for paraxylene. This is clearly illustrated by the values given in the patents [16]. At the same time, diffusion processes are influenced by the cations as will be illustrated below.

- The Si/Al ratio is also important as demonstrated by Seko et al. [17]. According to these results, para-xylene selectivity is increasing with increasing $\mathrm{Si} / \mathrm{Al}$ ratio in the range 1.2 to 3 .

Therefore, it clearly appears that the cation size, number and positions strongly influence the adsorption and diffusion properties. It will now be shown how the neutron instrumentation can bring information on the different molecular motions, starting first with the vibrations.

The vibrational modes of the zeolite framework and of adsorbed molecules can be measured by inelastic 
neutron scattering [18]. Compared with infrared and Raman spectroscopies, INS has unique properties such as:

- the lack of selection rules;

- a high sensitivity towards hydrogen atoms so that an adsorbed molecule will give a larger signal than the zeolite;

- a clear understanding of the different factors which govern the scattered intensities.

If the zeolite contains few protons, the spectrum of the zeolite will have a low intensity whereas one may have only limited frequency windows in infrared or the zeolite may fluoresce in the laser beam in Raman.

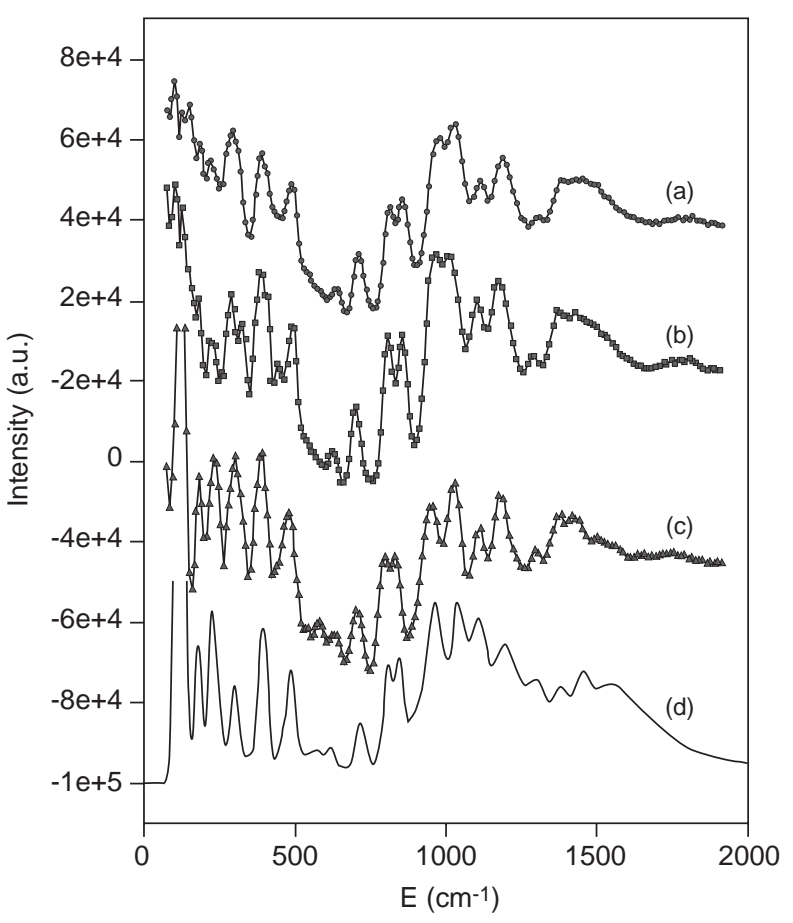

Figure 4

Vibrational spectra obtained for the para-xylene molecule. a) in $\mathrm{NaX}$ (loading of 3 molecules per supercage); b) in $\mathrm{BaX}$ (same loading as in $\mathrm{NaX}$ ); c) in the solid phase; d) simulated spectrum for solid para-xylene (the spectra were measured at $5 \mathrm{~K}$ on the IN1BeF spectrometer of the ILL).

A calculation of the INS spectrum of para-xylene, following a method described in ref. 19, is shown in Figure 4d. At low energy transfers, the fundamentals are the most intense features in the spectrum. However, overtones and combinations tend to dominate the spectrum at large energy transfers, because large scattering vectors $\mathbf{Q}$ are implied on a BeF spectrometer. The Debye-Waller factor decreases the intensity of the fundamentals as $\mathbf{Q}$ increases, Eq. (8), and the intensity is redistributed into multiphonon terms [19]. The simulated INS spectrum, Figure $4 d$, is in reasonable agreement with the spectrum measured for bulk para-xylene, Figure 4c. The strongest peaks are those which involve large displacement vectors for the $\mathrm{H}$ atoms, Eq. (8). $\mathrm{CH}_{3}$ bendings will thus be more intense than ring torsions. The largest intensity is obtained for the methyl torsions, at $120 \mathrm{~cm}^{-1}$, because of the large hydrogen atoms displacements and also because of the better resolution of the spectrometer at low energies.

The spectra reported in Figure 4 for adsorbed para-xylene were obtained after subtraction of the contribution from the degassed zeolites. The scattering from the molecule is much larger than the signal of the zeolite because of the large incoherent cross-section of hydrogen. There are clear differences between the spectra obtained in $\mathrm{NaX}$ and $\mathrm{BaX}$ zeolites with the solid phase, especially at small energy transfers (similar results were obtained for meta-xylene). It appears that for the adsorbed molecules, the methyl torsions are strongly perturbed, due to interactions with the oxygen atoms of the zeolite. A simulation of the spectra measured for the adsorbed xylenes would require as inputs the adsorption geometry of the molecules and a force field describing the sorbate, the sorbate-sorbate and the sorbate-framework interactions.

The rotational motions of para- and meta-xylene in $\mathrm{NaX}$ and $\mathrm{BaX}$ zeolites were measured using a TOF spectrometer [20]. The maximum energy transfer is now much smaller: 1.5 meV (Fig. 5), instead of hundreds of meV (Fig. 4) so that transitions between vibrational levels are not excited. The timescales accessible on a TOF instrument are longer than on a beryllium-filter spectrometer (Fig. 1). QENS spectra obtained for para-xylene in the two zeolites, at the same loading and temperature, are shown in Figure 5. The spectra consist of a narrow elastic component superimposed on a broader quasi-elastic component. From the series of spectra obtained at the different $Q$ values, one can conclude that only rotational motions are observed on the timescale of the TOF experiment $(1-100 \mathrm{ps})$, in both zeolites. The width of the elastic peak is given by the instrumental resolution, which means that the diffusion between supercages is too slow to be measured on this TOF spectrometer. A spectrum measured for liquid para-xylene on the same spectrometer is shown in Figure 6. Although the spectrum of the liquid was obtained at a lower temperature $(300 \mathrm{~K})$ and at a smaller $Q$ value, compared with the adsorbed molecules (Fig. 5), the much larger broadening reflects the fast isotropic rotations and translations. The narrowing observed when the molecules are adsorbed in the zeolites is a clear example of a confinement effect: the dynamics are considerably slowed down.

As formulated in Eq. (10), QENS spectra corresponding to a rotational motion can be resolved into two contributions: an elastic component, whose intensity is given by $A_{0}(\mathbf{Q})$ : the EISF, and a quasi-elastic contribution which consists in a sum of Lorentzian functions. The EISF is determined experimentally from the ratio of the purely elastic intensity to the 

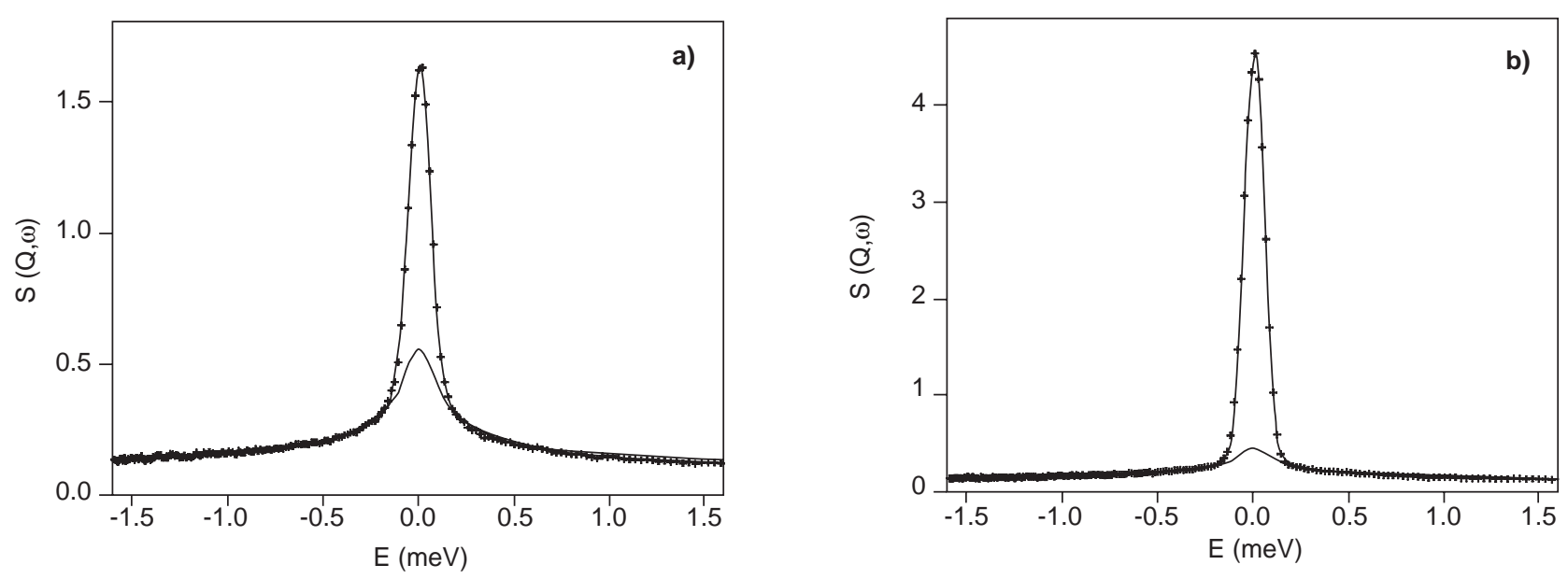

Figure 5

Comparison between experimental (+) and calculated (full line) QENS spectra obtained on a TOF instrument for para-xylene in: a) NaX, and b) BaX zeolites ( $\mathrm{T}=460 \mathrm{~K}$, loading: 3 molecules per supercage, $\mathrm{Q}=2 \AA^{-1}$ ). The separation between the elastic and quasi-elastic components of the rotation is indicated.

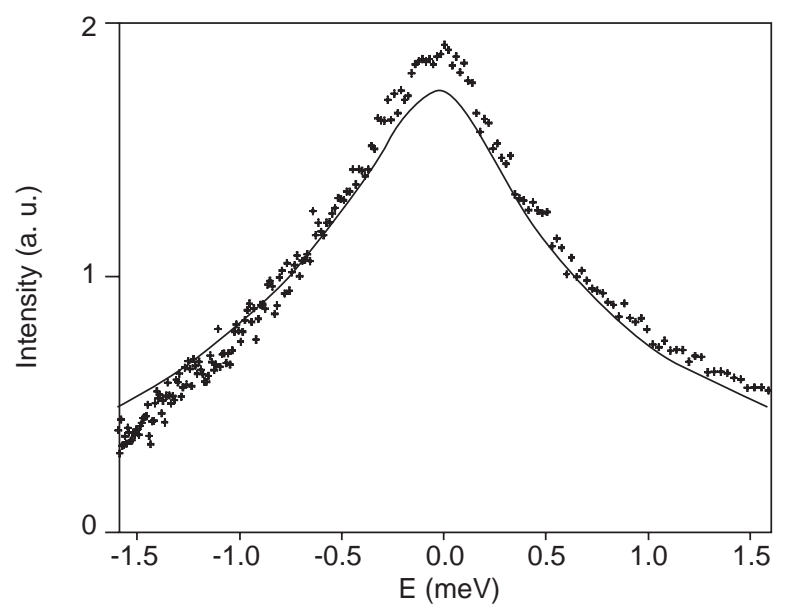

Figure 6

QENS spectrum measured for liquid para-xylene, at $300 \mathrm{~K}$ $\left(\mathrm{Q}=1.53 \AA^{-1}\right)$. The solid line is calculated from a convolution between translation and an approximate rotational model (isotropic rotation on a sphere) [20].

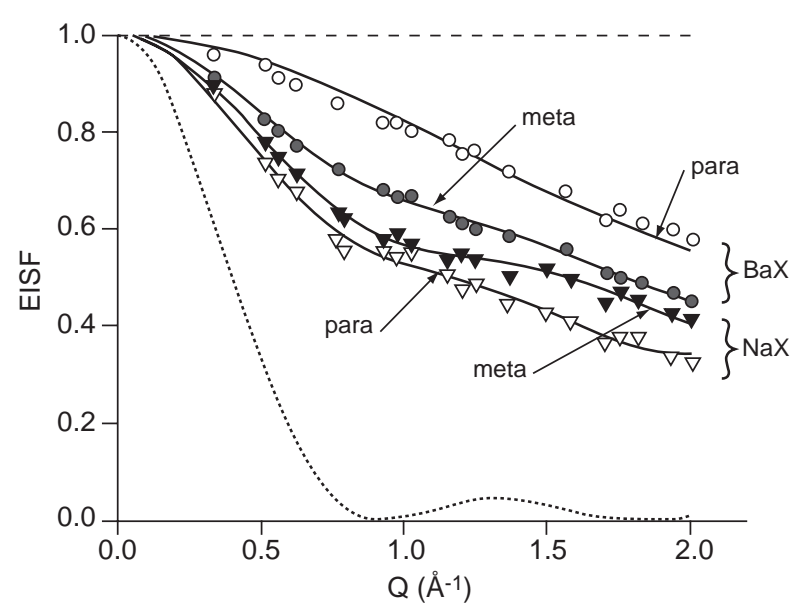

Figure 7

Experimental and calculated EISF values: $(\bigcirc)$ para-xylene in $\mathrm{BaX},(-)$ meta-xylene in $\mathrm{BaX},(\boldsymbol{\nabla})$ meta-xylene in $\mathrm{NaX}$, $(\nabla)$ para-xylene in $\mathrm{NaX}(\mathrm{T}=460 \mathrm{~K}$, loading: 3 molecules per supercage, on average). The dotted line corresponds to an isotropic rotation on a sphere of radius $3.4 \AA$, the dashed line to immobile molecules. total intensity (elastic + quasi-elastic). The values obtained at the different $Q$ values can be compared on the same graph since the scattering function for rotations is normalised

$$
\int S_{i n c}^{r o t}(Q, \omega) d \omega=1
$$

EISF values obtained at $460 \mathrm{~K}$ for the two isomers in the two zeolites are reported in Fig. 7. If all the hydrogen atoms of a xylene molecule would be immobile, on the timescale of the experiment, the EISF would be constant and equal to 1 (the dashed line in Fig. 7). If the molecules would be performing isotropic rotation, like in the liquid, the EISF would drop very quickly (the dotted line in Fig. 7). Therefore, the EISF for the adsorbed molecules is an intermediate case. For a given $Q$ value, a smaller EISF means that the spatial distribution of a xylene molecule is larger. It appears from Figure 7 that larger amplitude motions are observed for both xylenes in $\mathrm{NaX}$, compared with $\mathrm{BaX}$. This is also evident from Figure 5: the quasi-elastic component is larger in Figure 5a, corresponding to para-xylene in $\mathrm{NaX}$, 
than in Figure 5b, in BaX. Furthermore, one can conclude from Figure 7 that para-xylene is more strongly blocked than meta-xylene in $\mathrm{BaX}$, but the reverse situation is found in $\mathrm{NaX}$.

The simulation of the EISF data (full lines in Fig. 7) indicates that for para-xylene in BaX, only the two methyl groups are rotating, in the temperature range $295-460 \mathrm{~K}$. For meta-xylene in $\mathrm{BaX}$, there are oscillations of the molecules around their pseudo- $\mathrm{C}_{6}$ axis, in addition to the rotation of the methyl groups. The amplitude of oscillation $\Delta \phi$ varies between $8^{\circ}$ at $295 \mathrm{~K}$, to $11^{\circ}$ at $460 \mathrm{~K}$. In NaX zeolite, the methyl groups of the two xylene isomers are also rotating, but the amplitudes of oscillation of the ring are larger than in $\mathrm{BaX}: \Delta \phi$ is $20^{\circ}$ for para-xylene and $14^{\circ}$ for meta-xylene, at $460 \mathrm{~K}$. These results imply a stronger interaction between the $\pi$-electron density of the aromatic rings and the Ba cations than with the Na cations.

In order to observe diffusion, occurring at longer timescales, a higher energy resolution is needed. A backscattering (BS) spectrometer provides a resolution about 100 times better than a TOF instrument (Fig. 1). Some of the spectra measured for para-xylene in $\mathrm{NaX}$ and $\mathrm{BaX}$ zeolites on a BS spectrometer are shown in Figure 8 [20]. The maximum energy transfer is now $10 \mu \mathrm{eV}$, instead of $1.5 \mathrm{meV}$ on the TOF instrument. One is thus probing a very narrow energy range of Figure 5, the one concerning the elastic peak region. This means that one is not sensitive anymore to the quasi-elastic broadening due to the rotations.

A comparison of the spectra obtained at the same temperature and the same $Q$ value in $\mathrm{NaX}$ and $\mathrm{BaX}$ zeolites, Figure 8 , shows that the broadening, hence the diffusivity, is quite different in the two zeolites. In NaX zeolite, all the spectra obtained at the different $Q$ values could be fitted with a
Lorentzian function, due to diffusion between supercages, convoluted with the instrumental resolution. An example is shown in Figure 8a: the dashed line corresponds to a diffusion motion, an additional elastic intensity is fitted to take into account the variations of Bragg peaks intensities after adsorption [20]. The $H W H M$ of the Lorentzian functions obtained from fitting individually the spectra of para-xylene in $\mathrm{NaX}$ at a given loading and temperature are plotted in Figure 9 as a function of $Q^{2}$. The error bars are about $30 \%$ for the two lowest $Q$ values, they reach $50 \%$ for several other spectra because of the presence of Bragg contribution. All the spectra could be fitted simultaneously using a jump diffusion model. As mentioned in paragraph 2.2, the diffusion coefficient is essentially derived from the low $Q$ range, where one has a linear variation between the $H W H M$ and $Q^{2}$, Eq. (19). The diffusivities of the two isomers are in the range $10^{-10}-10^{-11} \mathrm{~m}^{2} \mathrm{~s}^{-1}$, para-xylene diffusing $10-25 \%$ faster than meta-xylene [20].

The QENS profiles measured in BaX are completely different from the ones observed in NaX, Fig. 8. Intercage diffusion cannot be measured in $\mathrm{BaX}$ on a $\mathrm{BS}$ spectrometer, even at the highest studied temperature of $550 \mathrm{~K}$. The spectra were fitted with a model involving jumps, within a supercage, between the four Ba cations [20].

The lower diffusivities of xylene isomers in $\mathrm{BaX}$ zeolite could be determined by the neutron spin echo (NSE) technique [21]. For this method, deuterated molecules are used as explained in paragraph 2.1 so that one obtains transport diffusivities $D_{t}$. The transport diffusivity is defined as the proportionality factor between the flux and a concentration gradient (Fick's first law). From equilibrium MD simulations, one obtains the corrected diffusivity $D_{0}$. These two diffusivities
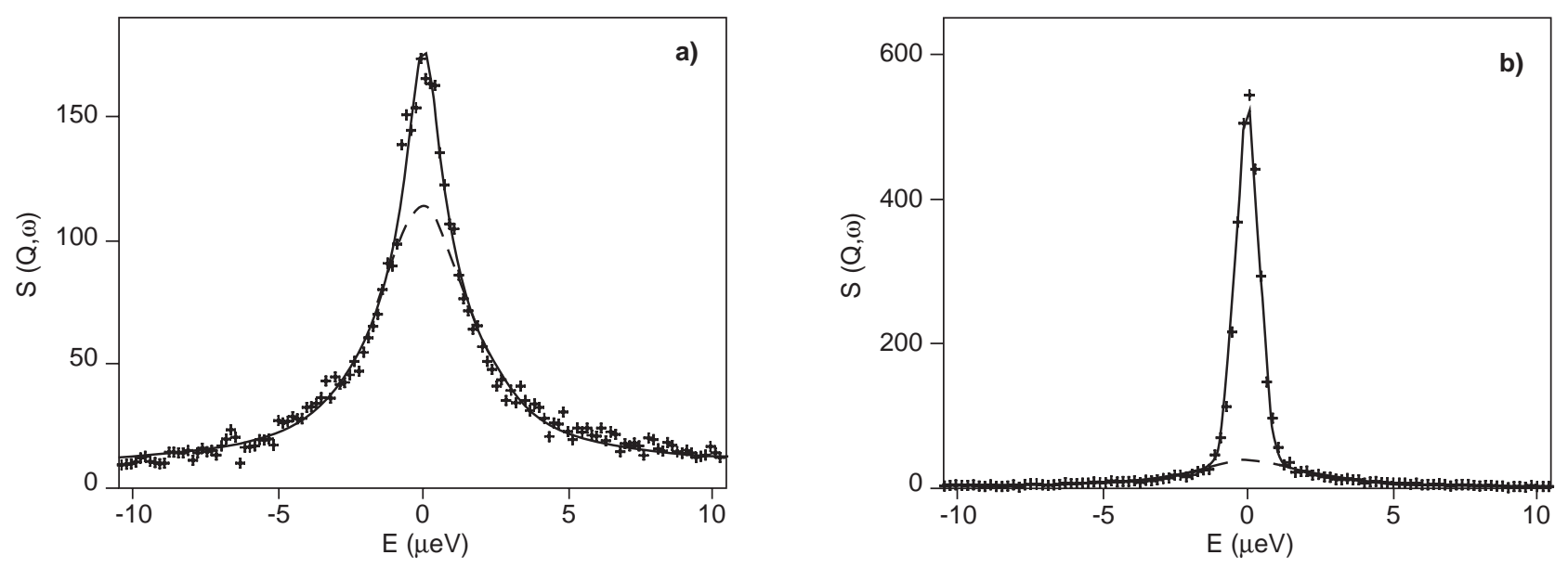

Figure 8

Comparison between experimental (+) and calculated (full line) QENS spectra obtained on a BS instrument for para-xylene in: a) NaX, and b) BaX zeolites ( $T=455 \mathrm{~K}$, loading: 3 molecules per supercage, $\left.\mathrm{Q}=0.65 \AA^{-1}\right)$. The solid line corresponds to the total intensity, the dashed line to the quasi-elastic contribution. 


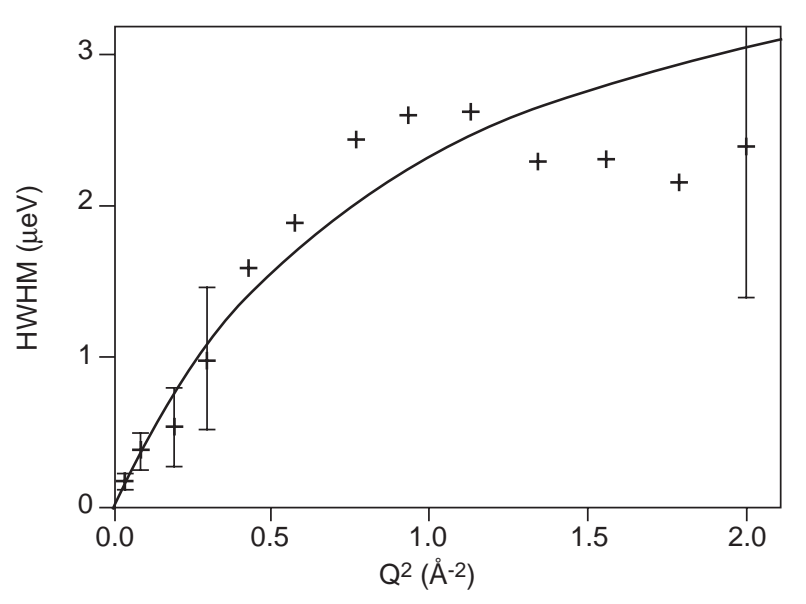

Figure 9

Broadenings derived for para-xylene in $\mathrm{NaX}$ zeolite $(\mathrm{T}=$ $455 \mathrm{~K}$, loading: 2 molecules per supercage). The crosses correspond to individual fits of the spectra (some error bars are shown), the solid line to a simultaneous fit with all spectra.

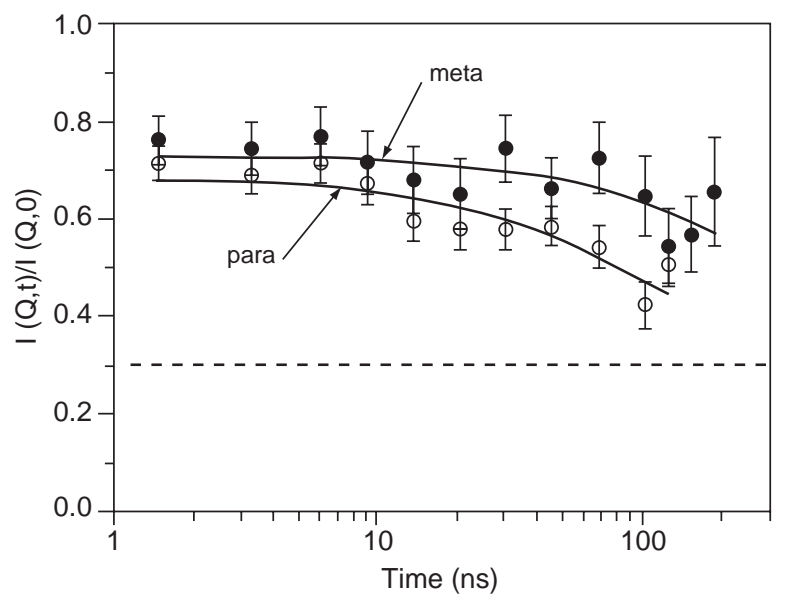

Figure 10

Normalised intermediate scattering functions obtained for deuterated xylenes in $\mathrm{BaX}$ zeolite: $(O)$ para-xylene, (O) meta-xylene $\left(\mathrm{T}=560 \mathrm{~K}, \mathrm{Q}=0.3 \AA^{-1}\right.$, loading: 2 molecules per supercage). The dashed line corresponds to the contribution from the zeolite and from the aluminium container [21]. are linked by considering that the driving force for diffusion is the chemical potential gradient, and not the concentration gradient:

$$
D_{t}(c)=D_{o}(c)\left(\frac{d \ln p}{d \ln c}\right)
$$

where $\mathrm{d} \ln p / \mathrm{d} \ln c$ is the thermodynamic factor. One approximation which is found in the literature (the Darken approximation) is to replace the corrected diffusivity in Eq. (24) by the self-diffusivity. However, the self, transport, and corrected diffusivities are only equal at zero concentration. All these diffusivities can have a different concentration dependence.

During a NSE experiment, data are recorded as a function of time, and not as a function of energy transfer as it is the case on TOF or BS spectrometers. One measures the intermediate scattering function, Eq. (11). The values obtained for the two xylene isomers are shown in Figure 10, at a given temperature and $Q$ value (only one loading corresponding to 2 molecules per supercage could be studied in the allocated time on this highly demanded instrument). Times up to 200 ns could be reached with the new NSE instrument at the ILL. For a diffusive process, one expects an exponential decay with time

$$
\frac{I(Q, t)}{I(Q, 0)}=\exp \left(-D Q^{2} t\right)
$$

A simple visual inspection of Figure 10 indicates that the diffusion coefficient of para-xylene is larger than the one of meta-xylene, the decay being faster. This was found to be true at all studied temperatures [21]. From measurements at two different $Q$ values $\left(0.2\right.$ and $\left.0.3 \AA^{-1}\right)$, and using a jump diffusion model, the diffusivities of the two isomers could be derived. At $560 \mathrm{~K}$, the diffusion coefficient of para-xylene is $8.7 \times 10^{-13} \mathrm{~m}^{2} \mathrm{~s}^{-1}$, whereas the one of meta-xylene is $2.7 \times 10^{-13} \mathrm{~m}^{2} \mathrm{~s}^{-1}$. It appears that the diffusivities in $\mathrm{BaX}$ are more than 2 orders of magnitude smaller than in $\mathrm{NaX}$. The activation energies for diffusion in BaX are $29 \mathrm{~kJ} / \mathrm{mol}$ for para-xylene and $23.5 \mathrm{~kJ} / \mathrm{mol}$ for meta-xylene. These values are much larger than those in $\mathrm{NaX}$ since the activation energies of the two isomers in this zeolite are of $9.4 \mathrm{~kJ} / \mathrm{mol}$, at the same loading. It is interesting to note that the difference between the diffusivities of xylene molecules is much larger in $\mathrm{BaX}$ than in $\mathrm{NaX}$ : the para-isomer diffuses about 3 times faster than the meta-isomer in BaX, compared with only $10-25 \%$ faster in $\mathrm{NaX}$. This suggests that diffusion can play a role in the separation process, depending on operating conditions.

\subsection{Alkanes in MFI}

The structure type code MFI refers to ZSM-5 and to its microporous silica polymorph, silicalite. Silicalite, which has no compensating cations, is an ideal system for molecular simulation studies. The unit cell of silicalite contains 96 tetrahedral $\left(\mathrm{SiO}_{2}\right)$ units. The unit cell contains four straight channel sections and four zigzag channels with four channel intersections. The cross section of the zig-zag channels is elliptical while the straight channels have a nearly circular cross section of free diameter $0.54 \mathrm{~nm}$. The length of the straight section is equal to the cell constant $b(\sim 1.98 \mathrm{~nm})$ while 
$\mathrm{a}$ and $\mathrm{c}$ dimensions are $2.006 \mathrm{~nm}$ and $1.336 \mathrm{~nm}$, respectively. The framework density is about $1.8 \mathrm{~g} / \mathrm{cm}^{3}$ and the specific micropore volume is about $0.19 \mathrm{~cm}^{3} / \mathrm{g}$. These properties affect the desorption/diffusion characteristics of the zeolite. In siliceous zeolites the main sources of the adsorbent-adsorbate interactions are dispersion force interactions.

Silicalite is of great industrial interest as its adsorption properties allows to separate molecules according to the molecular sieving. The separation of branched isomers from linear ones is one of the main applications of this type of material for octane upgrading. This zeolite may be used either as a membrane or as an adsorbent but the accurate control of the separation requires a deep knowledge of diffusion processes in the pores of the zeolite.

Various techniques have been used to measure the diffusivities of linear alkanes in this zeolite (Fig. 11). The QENS measurements for $\mathrm{C}_{1}-\mathrm{C}_{6}$ were performed with a TOF spectrometer, a $\mathrm{BS}$ instrument was used for $\mathrm{C}_{8}-\mathrm{C}_{14}$ [22]. The diffusion of longer alkanes could be studied in the future on a NSE machine. The QENS experiments performed so far concerned only hydrogenated molecules. Therefore, it is the selfdiffusivities which are plotted in Figure 11 for QENS, as well as in the case of PFG NMR and simulations techniques. The values reported for the macroscopic techniques are corrected diffusivities derived from the measured transport diffusivities. Since all results were obtained at low loadings, one can safely compare the different diffusivities.

The agreement between QENS [22] and PFG NMR data [23] is reasonable. The same ZSM-5 sample was used for methane [24], but crystals of a different origin were used for longer alkanes. Recent measurements indicate real differences between the two techniques, due to the non-ideal crystallinity of MFI samples and to the different space scales accessible by the two techniques: the QENS measurements are unaffected by the existence of intracrystalline transport barriers (diffusion path of a few nanometers), whereas in PFG NMR the molecules have to cross internal interfaces between different regions within the crystal (diffusion path $\approx 1 \mu \mathrm{m}$ ). The importance of defects and of the $\mathrm{Si} / \mathrm{Al}$ ratio is highlighted by the new QENS value reported in Figure 11 for $\mathrm{C}_{8}$ in silicalite: the diffusivity is 4 times larger than in NaZSM-5.

The very large diffusivities predicted by MD simulations [25] were obtained at infinite dilution with a rigid framework so that thermalisation was not effective. Recent MD simulations are in much better agreement with experiment [30]. The trend found by a hierarchical simulation based on Brownian motion and transition state theory [26] is in better agreement with the QENS values, especially considering the new value obtained for $\mathrm{C}_{8}$ in silicalite.

The comparison with the single-crystal membrane results [27] shows a good agreement for short and long chains, but a large gap is noticeable in between. The ZLC (zero-length column) diffusivities [28] are systematically 2 or 3 orders of magnitude lower than the QENS values.

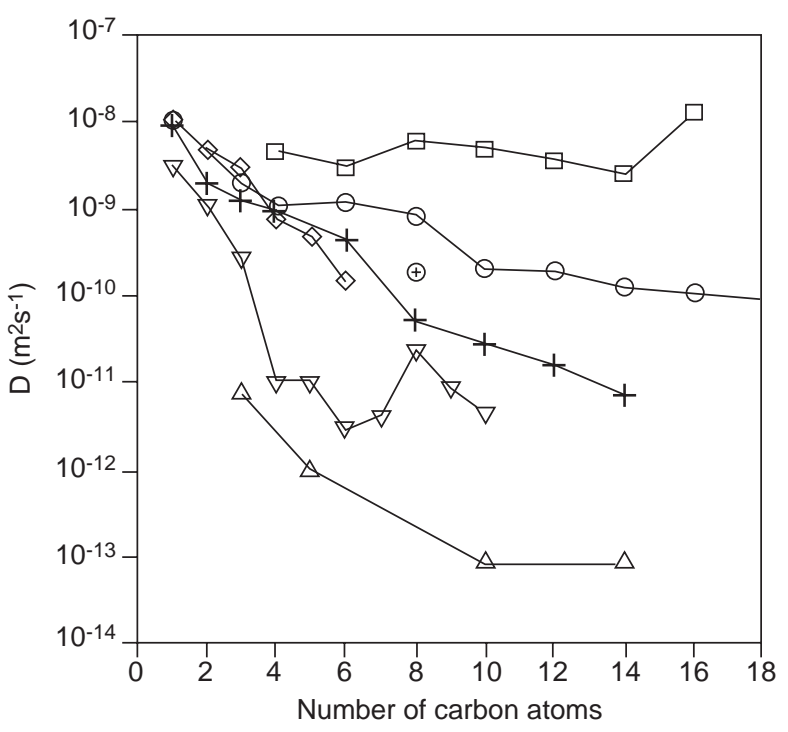

Figure 11

Diffusion coefficients of $n$-alkanes obtained at $300 \mathrm{~K}$ by various experimental and theoretical methods in MFI-type zeolites: $(\square)$ MD simulations [25], (O) hierarchical approach [26], (+) QENS [22], $(\diamond)$ PFG NMR [23], ( $\nabla)$ single-crystal permeation [27], and $(\triangle)$ zero-length column (ZLC) [28]. The QENS values were obtained in a NaZSM-5 sample with a $\mathrm{Si} / \mathrm{Al}$ ratio of 32 , in silicalite the diffusivities of the longer n-alkanes are larger, e.g. $(\oplus)$

The diffusion of branched alkanes has been less studied. QENS measurements performed on a BS spectrometer on hydrogenated isobutane gave diffusivities in the range $2-5 \times 10^{-12} \mathrm{~m}^{2} \mathrm{~s}^{-1}$, for temperatures between 450 and $570 \mathrm{~K}$ [29]. A reasonable agreement was observed with the supported membrane technique [29]. However, the QENS broadenings were small, only $10-20 \%$ of the instrumental resolution. More recent measurements were performed on a NSE instrument, using deuterated isobutane. The transport diffusivity derived at $490 \mathrm{~K}$ is of $2 \times 10^{-11} \mathrm{~m}^{2} \mathrm{~s}^{-1}$, which is 7 times larger than the self-diffusivity previously obtained in NaZSM-5. Part of this difference is due to the thermodynamic factor, Eq. (24), but a blocking effect of the Na cations is also probably observed.

\section{3 n-Alkanes in 5A Zeolite}

LTA zeolite is one of the best known zeolitic structures and it is part of numerous adsorption processes in the field of refining, petrochemicals or natural gas treatment. One of its most extensively used properties is the ability of the calcium form (5A) to adsorb linear alkanes while rejecting branched ones. This property is devoted to the production of highly branched $\mathrm{C}_{5}-\mathrm{C}_{6}$ paraffins for octane upgrading in gazoline or for supplying linear $\mathrm{C}_{10}-\mathrm{C}_{14}$ alkanes for linear alkylbenzene synthesis. 


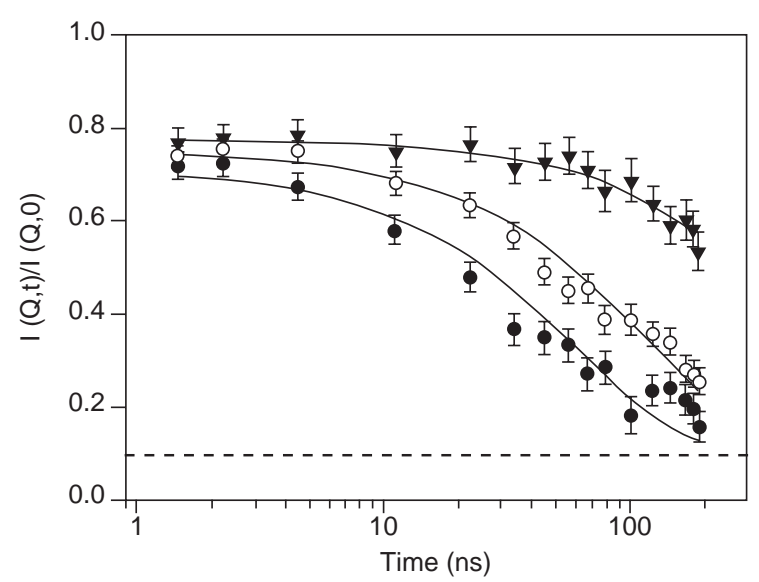

Figure 12

Normalised intermediate scattering functions obtained for some $n$-alkanes in $5 \mathrm{~A}$ zeolite, at $475 \mathrm{~K}:(\bullet) \mathrm{C}_{12},(\bigcirc) \mathrm{C}_{10}$, and $(\boldsymbol{\nabla}) \mathrm{C}_{8}\left(\mathrm{Q}=0.2 \AA^{-1}\right)[31]$.

For all these applications, mass transfer properties of $n$-alkanes in 5A zeolite is a key parameter as it directly influences the productivity of the sieve, that is to say the size of the unit and the operating costs. These mass transfer properties are related to the structure of the zeolite which may be influenced by preparation conditions and in particular by the calcination temperature and atmosphere. For these reasons, it is important to accurately control the synthesis of the zeolite and to develop fine tools for the measurement of the diffusion coefficients in the crystals.

The diffusion of $n$-alkanes in $5 \mathrm{~A}$ zeolite is too slow to be measured on TOF spectrometers, it is at the limit of the resolution of BS instruments, but it is easily accessible by the NSE technique [31]. Normalised intermediate scattering functions measured in the same experimental conditions for three $n$-alkanes in $5 \mathrm{~A}$ zeolite, at $475 \mathrm{~K}$, are shown in Figure 12. Since a faster decay corresponds to a larger diffusion coefficient, Eq. (25), a mere look at Figure 12 leads to the conclusion that the diffusivity increases with increasing chain length, in the sequence $\mathrm{C}_{8}<\mathrm{C}_{10}<\mathrm{C}_{12}$. From the data obtained at two different $Q$ values and using a jump diffusion model, diffusion coefficients could be derived. These diffusivities are compared in Figure 13 with those reported in the literature in other zeolite structures. In NaX and ZSM-5, a monotonous decrease in the self-diffusion coefficients is observed with increasing chain length. From the NSE experiments performed in $5 \mathrm{~A}$, one finds that the transport diffusivity drops to a minimum at $\mathrm{C}_{8}$, rises for $\mathrm{C}_{10}$, has a maximum at $\mathrm{C}_{12}$, and then decreases again for $\mathrm{C}_{14}$. Since the loading was selected such that the number of carbon atoms per cavity stays constant (i.e. $12 \mathrm{C}$ per $\alpha$-cage), a similar maximum is expected for the corrected diffusivity, after correcting from the thermodynamic factor. The activation energy is larger for $\mathrm{C}_{8}, 41 \mathrm{~kJ} / \mathrm{mol}$, than for $\mathrm{C}_{12}, 35 \mathrm{~kJ} / \mathrm{mol}$.

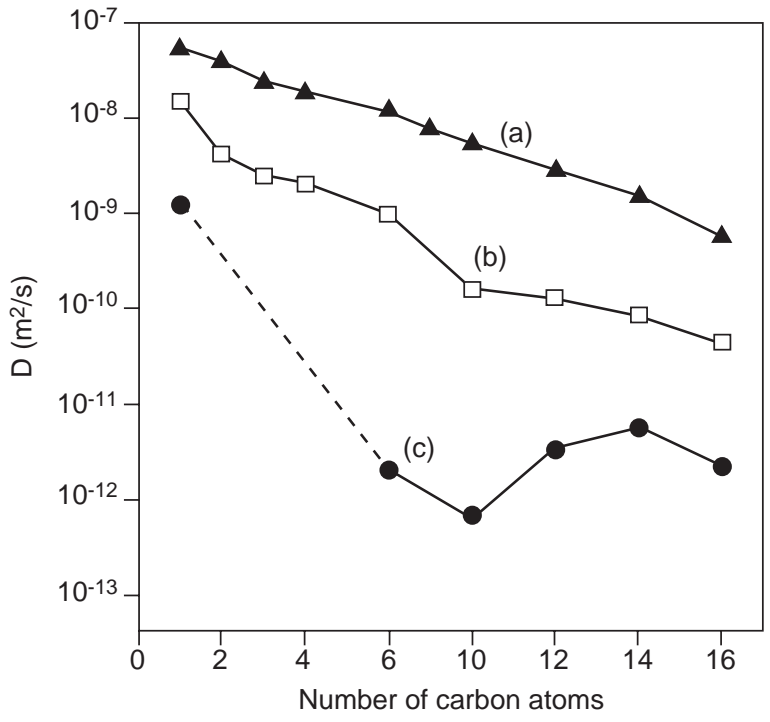

Figure 13

Diffusion coefficients of linear alkanes in various zeolites, obtained by different techniques at $475 \mathrm{~K}$, as a function of the carbon number: a) self-diffusivities derived from PFG NMR in NaX; b) self-diffusivities measured by QENS in ZSM-5; c) transport diffusivities obtained by NSE in 5A zeolite [31].

The maximum of diffusivity, as well as the minimum in the activation energy, observed for $\mathrm{C}_{12}$ in $5 \mathrm{~A}$ zeolite can be explained by the 'window effect'. This term was coined by Gorring to interpret anomalous transport results obtained in zeolite $\mathrm{T}$ (an offretite/erionite intergrowth), where he found that molecules in the $\mathrm{C}_{12}$ range diffused about 2 orders of magnitude faster than those in the $\mathrm{C}_{8}$ range [32]. This concept was developed to designate the higher transmittance of the erionite cages for molecules of a given critical length. However, the experimental conditions used by Gorring have been criticized, and since no other group could reproduce his periodic variation in diffusivity, the existence of this window effect is still disputed, although some recent molecular simulations predict its occurrence in various zeolite structures. A schematic interpretation of the NSE results is shown in Figure 14: a $C_{8}$ molecule fits well into a cavity, whereas $C_{12}$ is too long to sit comfortably in a cage and adopts a conformation where one end of the molecule extends through a window. The energy barrier (largely entropic) is thus easier to cross for the longer molecule, leading to a higher diffusivity and to a lower activation energy.

\subsection{Water in A- and X-Type Zeolites}

It is well established that preadsorbed water can modify the adsorption properties of xylenes in $\mathrm{BaX}$ zeolite as well as of paraffins in 5A zeolite. This is illustrated by the work of Furlan et al. who put into evidence a maximum in the 


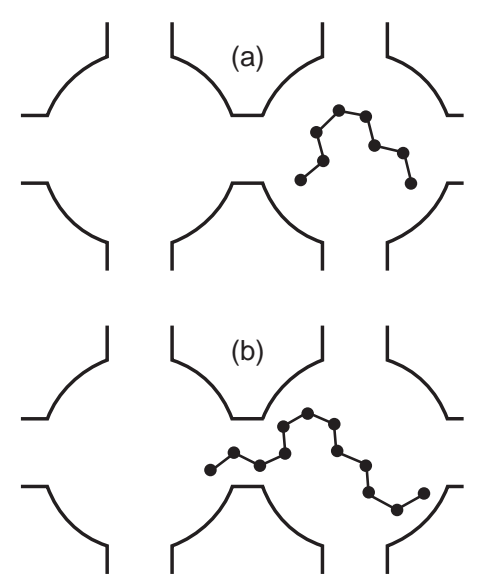

Figure 14

Schematic representation of the configuration of two $n$-alkanes in $5 \mathrm{~A}$ zeolite: $\mathrm{C}_{8}$ fits well into a cage a); but one end of $\mathrm{C}_{12}$ sits in a window between two cages $\mathrm{b}$ ).

selectivity for paraxylene in $\mathrm{BaX}$ zeolite as a function of the water content [33]. It was demonstrated by Pichon et al. that the water molecules are strongly interacting with the cations and consequently weakening the interactions between the aromatic ring and the cation [34, 35]. It has also been shown by pulsed-field gradient NMR (PFG NMR) that low quantities of water have a large effect on the self-diffusion of unsaturated hydrocarbons [36].

As the presence of water is important for the adsorption selectivity, it is worth considering the dynamics of this preadsorbed molecule and its influence on the zeolite itself. The diffusion of water in 4A and 5A zeolites, and in BaX zeolite has recently been studied by QENS.
The self-diffusion of water in A-type zeolites has been jointly studied by incoherent QENS and PFG NMR [37]. Samples with various calcium content were prepared from a batch of $4 \mathrm{~A}\left(100 \% \mathrm{Na}^{+}\right)$zeolite of commercial origin (a sample where $61.5 \%$ of $\mathrm{Na}^{+}$has been replaced by $\mathrm{Ca}^{2+}$ will be designated as $5 \mathrm{~A}(61.5 \%)$. Scanning electron microscopy images of the material showed that the zeolite grains were rather small (diameter ranging from 0.25 to $4 \mu \mathrm{m}$ ), with a lot of defects under the form of stacking faults or twins. The QENS experiments were performed on a BS spectrometer. Some of the spectra obtained in $4 \mathrm{~A}$ and $5 \mathrm{~A}(61.5 \%)$ at similar temperatures for the same water concentration are shown in Fig. 15. The $Q$ value in Figure 15 is $0.29 \AA^{-1}$, and at this $Q$ value the broadening is essentially due to long-range diffusion. It appears therefore that the broadening, hence the selfdiffusivity, is larger in $4 \mathrm{~A}$ than in $5 \mathrm{~A}$ zeolite. When the calcium content increases, it is found that the water diffusivity decreases, at the same water loading. The first water molecules interact more strongly with the cations, and with increasing water content the overall mobility is expected to increase due to a reduced interaction with the zeolite. This is indeed what is found by both QENS and PFG NMR techniques, as shown in Figure 16. A larger difference between the diffusivities at loadings of 5 and 15 molecules per pseudo-unit cell was observed when the Ca content increases (due to the stronger interaction of water with calcium ions compared with sodium). At a higher water concentration, 25 molecules per pseudo-unit cell, the self-diffusivities decrease due to the reduced accessible volume. Though the QENS and PFG NMR data agree in their evidence with respect to the influence of loading and cation content on the water self-diffusivities, there are notable differences in the absolute values of the diffusivities and in the activation energies, $E_{a}$. It appears from Figure 16 that the QENS values are always above the PFG NMR values and that the slopes in
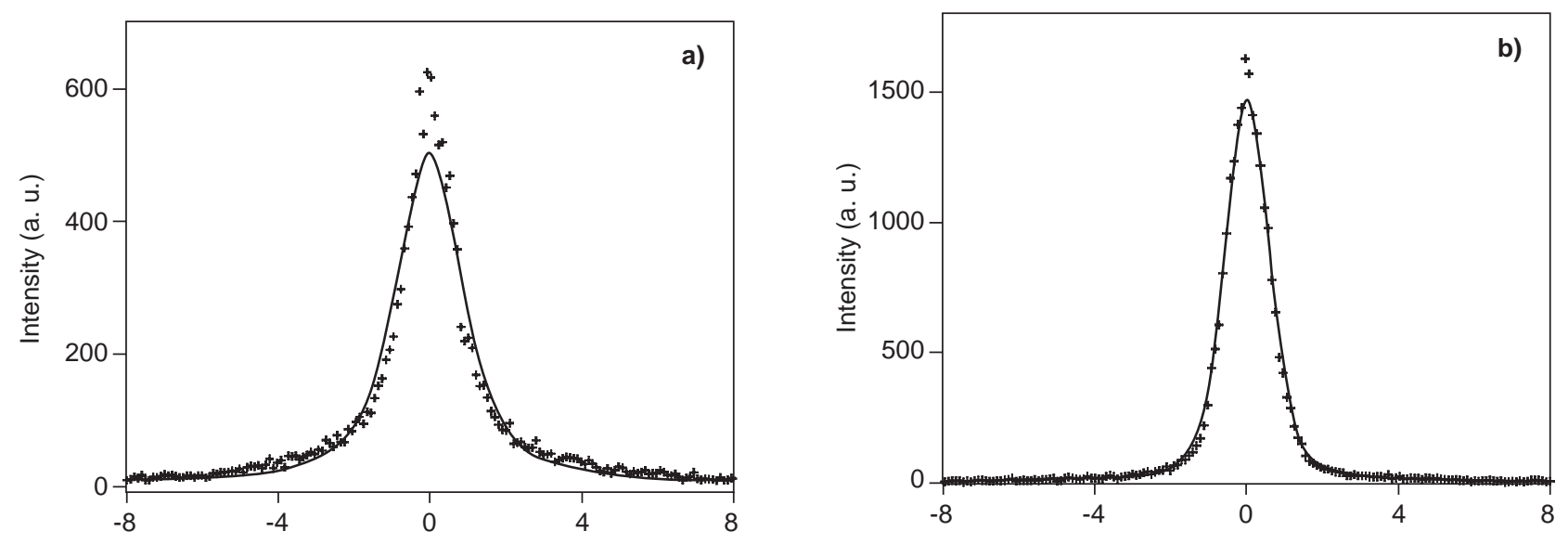

Figure 15

Comparison between experimental (+) and calculated (full line) QENS spectra obtained for $\mathrm{H}_{2} \mathrm{O}$ in: a) $4 \mathrm{~A}$ zeolite, $\mathrm{T}=430 \mathrm{~K}$; b) $5 \mathrm{~A}(61.5 \%$ ) zeolite, $\mathrm{T}=421 \mathrm{~K}$ (loading of 5 molecules per pseudo-unit cell, $\mathrm{Q}=0.29 \AA^{-1}$ ). 

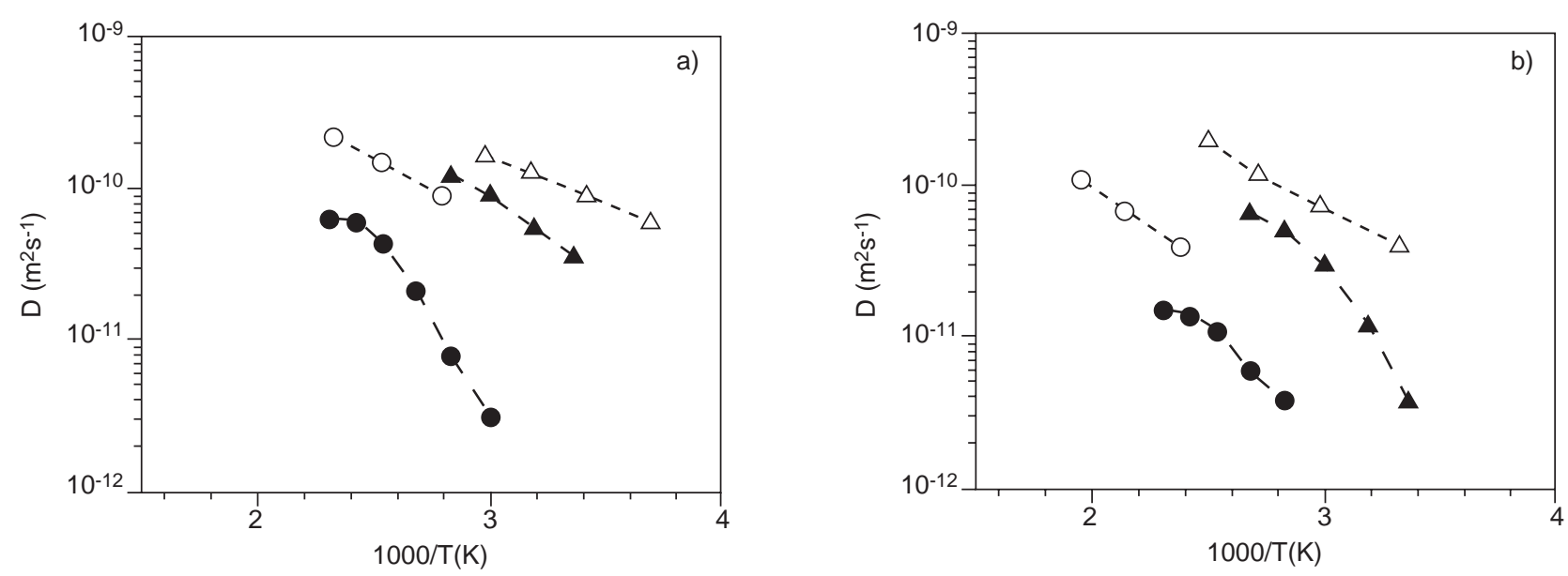

Figure 16

Arrhenius plots of the diffusivities of water in: a) 4A zeolite; b) 5A (61.5\%), measured by QENS (open symbols) and PFG NMR (filled symbols), at two different loadings: 5 molecules per pseudo-unit cell $(\bigcirc, \bullet)$, and 15 molecules per pseudo-unit cell $(\triangle, \mathbf{\Delta})$.

the Arrhenius plots (i.e. $\mathrm{E}_{\mathrm{a}}$ ) of the PFG NMR data are larger than those of the QENS data. The explanation has been given in paragraph 3.2 when comparing self-diffusivities of linear alkanes in MFI samples. The length of the diffusion path probed by QENS is smaller than the spacings between internal transport barriers. On the other hand, during the observation time of the PFG NMR experiment, the molecules have to pass through several internal boundaries between the individual ranges of ideal crystallinity. These boundaries require a larger energy of thermal activation to be overcome and lead simultaneously to lower diffusivities.

The mobility of water in $\mathrm{BaX}$ zeolite has been found to be much lower than in 5A zeolites [38]. For a loading of 15 molecules per supercage, a continuous evolution of intensity was observed at $440 \mathrm{~K}$, indicating a progressive slowing down of the mobility of water molecules. After $20 \mathrm{~h}$, the intensity measured on a BS spectrometer was only elastic, which implies that the diffusion coefficient is lower than $10^{-12} \mathrm{~m}^{2} \mathrm{~s}^{-1}$. This shows that after sample preparation: water adsorption at room temperature and equilibration at $375 \mathrm{~K}$, the sample was not in thermal equilibrium. Whereas in 5A zeolites, which were equilibrated at the same temperature, no variation in intensity was detected as a function of time. For a loading of 10 water molecules per supercage of $\mathrm{BaX}$, the QENS measurements indicate that a higher temperature, $520 \mathrm{~K}$, is needed to reach equilibrium. It should be noted that in incoherent QENS, one observes only the hydrogen atoms motions, so that changes in the dynamics of water could be accompanied by a redistribution of the Ba cations. For the lowest loading, 5 molecules per supercage, it appears that water molecules cannot reach their equilibrium positions, up to $520 \mathrm{~K}$. In order to determine the very low diffusivities of water in equilibrated $\mathrm{BaX}$ samples, one would have to recourse to the NSE technique.

\section{CONCLUSIONS}

Neutron scattering techniques were used to characterise intracrystalline diffusion in various hydrocarbon/zeolite systems and water/zeolite systems. These systems are devoted to adsorption separations in the field of refining and petrochemical industry. The zeolites have 8-, 10- and 12membered rings and various compensating cations.

Despite the complex behaviour of adsorbed phase dynamics, neutron techniques provided a good insight into the diffusion mechanisms, in a range of diffusivities from $10^{-8}$ to $10^{-13} \mathrm{~m}^{2} \mathrm{~s}^{-1}$. It revealed that diffusion plays an important role in all the separations which were studied.

For xylene in X-type zeolites, we demonstrated that the diffusion coefficients are strongly dependant upon the cation and that diffusion is slower for meta-xylene than for paraxylene in $\mathrm{BaX}$. In these systems, intracrystalline diffusion seems not to be rate limiting.

For alkanes in MFI zeolites, linear alkanes diffuse much faster than branched ones. The importance of the $\mathrm{Si} / \mathrm{Al}$ ratio was put into evidence: for example the diffusion of $n$-octane in silicalite is 4 times larger than in Na-ZSM-5.

For $n$-alkanes in 5A zeolite, diffusion coefficients were measured from $\mathrm{C}_{6}$ to $\mathrm{C}_{14}$. Our results showed that the diffusivities are increasing from $\mathrm{C}_{8}$ to $\mathrm{C}_{12}$ which is an unexpected result, and which is in line with the famous and controversial 'window effect'. The interpretation of this trend is based on the conformation of the molecules: $\mathrm{C}_{8}$ fits well into the cavity while $\mathrm{C}_{12}$ is too long.

The mobility of water in $\mathrm{BaX}$ zeolite is much lower than in $\mathrm{NaCaA}$ zeolites and it is more difficult to reach adsorption equilibrium. This illustrates the high interaction between water and the Ba cations, which reminds the larger interaction of xylenes with Ba cations, compared with Na cations. 


\section{REFERENCES}

1 Papadopoulos, G. K., Jobic, H. et Theodorou, D. N. (2004) J. Phys. Chem. B, 108, 12748-12756.

2 Jobic, H., Skoulidas, A. I. et Sholl, D. S. (2004) J. Phys. Chem. B, 108, 10613-10616.

3 Chandler, D. (1987) Introduction to Modern Statistical Mechanics, Oxford University Press, New York.

4 Hoogenboom, J. P., Tepper, H. L., van der Vegt, N. F. A. et Briels, W. J. (2000) J. Chem. Phys., 113, 6875-6881.

5 Bée, M. (1988) Quasielastic Neutron Scattering, Adam Hilger, Bristol.

6 Jobic, H., Kärger, J. et Bée, M. (1999) Phys. Rev. Lett., 82, 4260-4263.

7 Jobic, Molecular Sieves - Science and Technology, Vol. 7, Springer (in press).

8 Jobic, H., Tuel, A., Krossner, M., Sauer, J. (1996) J. Phys. Chem., 100, 19545-19550.

9 Jobic, H., Smirnov, K. S. et Bougeard, D. (2001) Chem. Phys. Lett., 344, 147-153.

10 Wilson, E. B., Decius, J. C. et Cross, P. C. (1955) Molecular Vibrations, Mc Graw-Hill, New York.

11 van Hove, L. (1954) Phys. Rev., 95, 249-262.

12 Mezei, F., éd. (1979) Neutron Spin Echo, Lectures Notes in Physics, Vol.128, Springer-Verlag, Berlin.

13 Jobic, H. (2000) in Recent Advances in Gas Separation by Microporous Membranes, Membrane Science and Technology Series 6, Kanellopoulos, N. K. (ed), Elsevier, p. 109-137.

14 Cook, J. C., Richter, D., Schärf, O., Benham, M. J., Ross, D. K., Hempelmann, R., Anderson, I. S. et Sinha S. K. (1990) J. Phys.: Condens. Matter, 2, 79-94.

15 Neuzil, R.W. (1971) U.S. Patent 3, 558, 730.

16 Stine, L. O. et Broughton, D. B. (1969) US Patent 3636121 to UOP.

17 Seko, M., Miyake, T. et Inada, K. (1980) Hydrocarbon Processing, Jan., 133.

18 Jobic, H. (2003) Curr. Opin. Solid State Mater. Sci., 6, 415422.

19 Jobic, H. et Lauter, H (1988) J. Chem. Phys., 88, 5450-5456.
20 Jobic, H., Bée, M., Méthivier, A. et Combet, J. (2001) Micropor. Mesopor. Mater., 42, 135-155.

21 Jobic, H., Méthivier, A. et. Ehlers, G. (2002) Micropor. Mesopor. Mater., 56, 27-32.

22 Jobic, H. (2000) J. Mol. Catal. A, 158, 135-142.

23 Heink, W., Kärger, J., Pfeifer, H., Datema, K. P. et Nowak, A. K. (1992) J. Chem. Soc. Faraday Trans., 88, 3505-3509.

24 Jobic, H., Bée, M., Caro, J, Bulow, M. et Kärger, J. (1989) J. Chem. Soc., Faraday Trans. 1, 85, 4201-4209.

25 Runnebaum, R. C. et Maginn, E. J. (1997) J. Phys. Chem. B, 101, 6394-6408.

26 Maginn, E. J., Bell, A. T. et Theodorou, D. N. (1996) J. Phys. Chem., 100, 7155-7173.

27 Talu, O., Sun, M. S. et Shah, D. B. (1998) AIChE J., 44, 681694.

28 Eic, M. et Ruthven, D. M. (1989) Stud. Surf. Sci. Catal., 49B, 897-905.

29 Millot, B., Méthivier, A., Jobic, H., Moueddeb, H. et Bée, M. (1999) J. Phys. Chem. B, 103, 1096-1101.

30 Leroy, F., Rousseau, B. et Fuchs, A. H., (2004) Phys. Chem. Chem. Phys. 6, 775-783.

31 Jobic, H., Méthivier, A., Ehlers, G., Farago, B. et Haeussler, W. (2004) Angew. Chem. Int. Ed., 43, 364-366.

32 Gorring, R. L. (1973) J. Catal., 31, 13-26.

33 Furlan, L. T., Chaves, B. C. et Santana, C. C. (1992) Ind. Eng. Chem. Res., 31, 1780.

34 Pichon, C., Méthivier, A. et Simonot-Grange, M. H. (2000) Langmuir, 16, 1931-1936.

35 Pichon, C., Méthivier, A. et Simonot-Grange, M. H. et Baerlocher, Ch. (2000) J. Phys. Chem. B, 103, 10197-10203.

36 Kärger, J. et Ruthven, D. M. (1992) Diffusion in Zeolites and Other Microporous Materials, John Wiley \& Sons, New York, p. 463.

37 Paoli, H., Méthivier, A., Jobic, H., Krause, C., Pfeifer, H., Stallmach, F. et Kärger, J. (2002) Micropor. Mesopor. Mater., 55, 147-158.

38 Jobic, H., Méthivier, A. et Seydel, T. (2005) C. R. Chimie, 8, (3-4).

Final manuscript received in April 2005 or distributed for profit or commercial advantage and that copies bear this notice and the full citation on the first page. Copyrights for components of this work owned by others than IFP must be honored. Abstracting with credit is permitted. To copy otherwise, to republish, to post on servers, or to redistribute to lists, requires prior specific permission and/or a fee: Request permission from Documentation, Institut français du pétrole, fax. +33147527078 , or revueogst@ifp.fr. 